удК 628

Н. С. Грибанова

Байкальский государственный университет, г. Иркутск, Российская Федерация

А. Ф. Шуплецов

Байкальский государственный университет, 2. Иркутск, Российская Федерация

\title{
ОСОБЕННОСТИ ПООПЕРАЦИОННОГО ПЛАНИРОВАНИЯ В КОНКУРЕНТНОЙ СРЕДЕ С ЦЕЛЬЮ ОПТИМИЗАЦИИ РЕСУРСНЫХ ПАРАМЕТРОВ ДЕЯТЕЛЬНОСТИ ПРОМЫШЛЕННОЙ КОМПАНИИ
}

\begin{abstract}
АНнотАция. Рыночные преобразования в России требуют адекватного изменения в механизмах управления компаниями и, в свою очередь, необходимо проведение революционного преобразования обслуживающих их информационных систем. Вакуум в технологии сбора и обработки управленческой информации заполняется адаптируемыми методиками планирования, формировавшиеся за рубежом в течение нескольких десятилетий. Зарубежные идеи на неподготовленной почве приживались с трудом или не приживались вовсе. Одной из современных характерной чертой в организации промышленного производства является преимущество мелкосерийного и индивидуального, в том числе позаказного производства, планирование учета затрат и калькулирование себестоимости продукции при быстро меняющемся портфеле заказов. Надобность раздельного вычисления отдельных операций по статьям затрат определена неоднородностью их нарастания и разным экономическим назначением. Автоматизация этих процессов занимает довольно длительный срок и крайне затратна.

кЛючЕВыЕ словА. Планирование; пооперационный метод; автоматизация бизнес процессов; управление; принятие решений.

ИНФОРМАЦИЯ О СТАТЬЕ. Дата Поступления 14 июня 2017 г.; дата принятия к печати 19 декабря 2017 г.; дата онлайн-размещения 29 декабря 2017 г.
\end{abstract}

N. S. Gribanova Baikal State University, Irkutsk, Russian Federation

A. F. Shupletsov Baikal State University, Irkutsk, Russian Federation

\section{FEATURES OF OPERATIONAL PLANNING IN A COMPETITIVE ENVIRONMENT IN ORDER TO OPTIMIZE THE RESOURCE PARAMETERS OF THE INDUSTRIAL COMPANY}

\begin{abstract}
Market reforms in Russia require an adequate change in the mechanisms for managing companies and, therefore, it is necessary to carry out a revolutionary reformation of their information systems. Vacuum in the collection technology and processing of management information is filled with adaptable planning methods that have been formed abroad for several decades. Foreign ideas on unprepared soil took root with difficulty or did not take root at all. One of the modern characteristic features in the organization of industrial production is the advantage of small-scale and individual businesses, including custom-made production, planning of cost accounting and calculating the prime cost of production with a rapidly changing portfolio of orders. The need for separate calculation of individual transactions by cost items is determined by the heterogeneity of their growth and different economic purposes. Automation of these processes takes quite a long time and is extremely costly.
\end{abstract}

\section{Baikal Research Journal}


KEYWORDS. Planning; operational method; automation of business processes; management; decision-making.

ARTICLE INFO. Received June 14, 2017; accepted December 19, 2017; available online December 29, 2017.

Если рассматривать количество промышленных компаний в России, то 90 \% являются компании малого и среднего бизнеса. Это со временем может измениться в сторону уменьшения. Разработка методологического подхода к решению задач производственного и финансового планирования, основанная на существующем технологическом процессе в секторе малого бизнеса является крайне актуальной. Целью работы является автоматизация движения финансовой информации в компании и создания базы перспективных финансовых решений для принятия управленческих мероприятий [1].

Автоматизация предметной области осуществляется в разных масштабах от единичных информационных процессов и процедур обработки задач управления до создания функционально полных автоматизированных информационных систем. Имеются разнообразные подходы к решению проблем автоматизации конкретной области: формирование, разработка автоматизированной информационной системы - постановка и автоматизация решения новых задач или комплекса задач; переход имеющихся задач на новый уровень информационных технологий автоматизации, связанных с преобразованием обработки данных; формирование автоматизации информационных систем в полном масштабе подсистем, функций и комплексов задач. Этот подход во многом определяет существование безлюдных производственных технологий [2, с. 75].

Методы экономико-математического моделирования дают возможность установить количественное выражение взаимосвязей между финансовыми показателями и их основными факторами; построить экономико-математическую модель по функциональной и корреляционной связи. Использование экономико-математических моделей позволяет быстро рассчитать несколько вариантов показателей и выбрать оптимальный план [3, с. 150].

Распространение мелкосерийной организации производства при быстрой смене портфеля заказов имеет определенные трудности. Во-первых, недостаточно регулярно выпускаются изделия, что в свою очередь, усложняет регулирование структуры производственных мощностей подразделений и производственной программы на определенный планово-учетный период времени. Во-вторых, необходимо уже на цеховом уровне производства предложить продукт, соответствующий заявленным потребительским требованиям, заданного стабильного качества и в обещанные сроки.

Возникает проблема совмещения чисто экономических критериев, свидетельствующих о доходности бизнеса, с одной стороны, и производственно-исполнительских критериев, определяющих расходы компании. С другой стороны минимизация времени выполнения заказов, максимизация загрузки оборудования и минимизация запасов.

На каких позициях будет формироваться теоретическая база постановки задачи исследования - это основная задача, которая выражается в описании методологических подходов к процессу автоматизации планирования и прогнозирования деятельности. Без решения вопроса на теоретическом уровне построения программы учета не представляется возможным.

Существующие программные продукты Российской разработки не решают этот вопрос, а лишь используют экономические наработки, принятые на сегодняшний день. Математическое моделирование производственных операций исследовано достаточно хорошо, моделирование в исследовании финансовых операций на основе пооперационного учета является практически неразработанной научной

\section{Baikal Research Journal}


проблемой. В основном эти расчеты ведутся на основе расчетов различных групп бюджетов компании.

Первыми к практическому решению этой проблемы подошли на Западе в результате разработки безлюдных технологий. Вторым этапом автоматизации встал вопрос формализации управленческого труда [4].

Подобные исследования в Советском союзе оставались чисто теоретическими и не имели выхода на практику, поскольку не был автоматизирован процесс производства на уровне безлюдных технологий. В связи с этим по большей части развивалась практическая составляющая процесса без исследования понятийной теоретической части в плане определений предмета исследования операций.

Идея методики пооперационного планирования как технологического, так финансового процессов состоит в пошаговом планировании времени наступления точек процесса и отслеживании фактического времени их завершения. Результат операции зависит от способа ее проведения, организации.

Последовательность работ разработки методики пооперационного планирования состоит в: разработке классификаторов событий, пошагового плана выполнения работ; построении плана на основе шаблона плана выполнения работ для определенной стадии производства.

Современным решением, обеспечивающим формирование пооперационных планов, является имитационное моделирование производства и становление технологий календарного планирования.

В настоящее время компьютеризация алгоритмов решения задач календарного планирования основывается на эвристических методах, в основном на схемах ветвления с функциями предпочтения [5, с. 174-186].

Сущность схемы ветвления в модели планирования производства состоит в следующем, после очередного встраивания в график работы актуализируется массив готовых к выполнению операций и выбирается из этого массива очередная из них в соответствии с правилами предпочтения.

Для совмещения традиционного калькулирования с методом пооперационного учета важно дополнить базовые формы с данными о движении промежуточных продуктов и изменении операционных заделов по каждой операции передела. Последовательность скоординированных шагов в процессе компьютерного моделирования прогнозных оценок представлена блок-схемой 1.

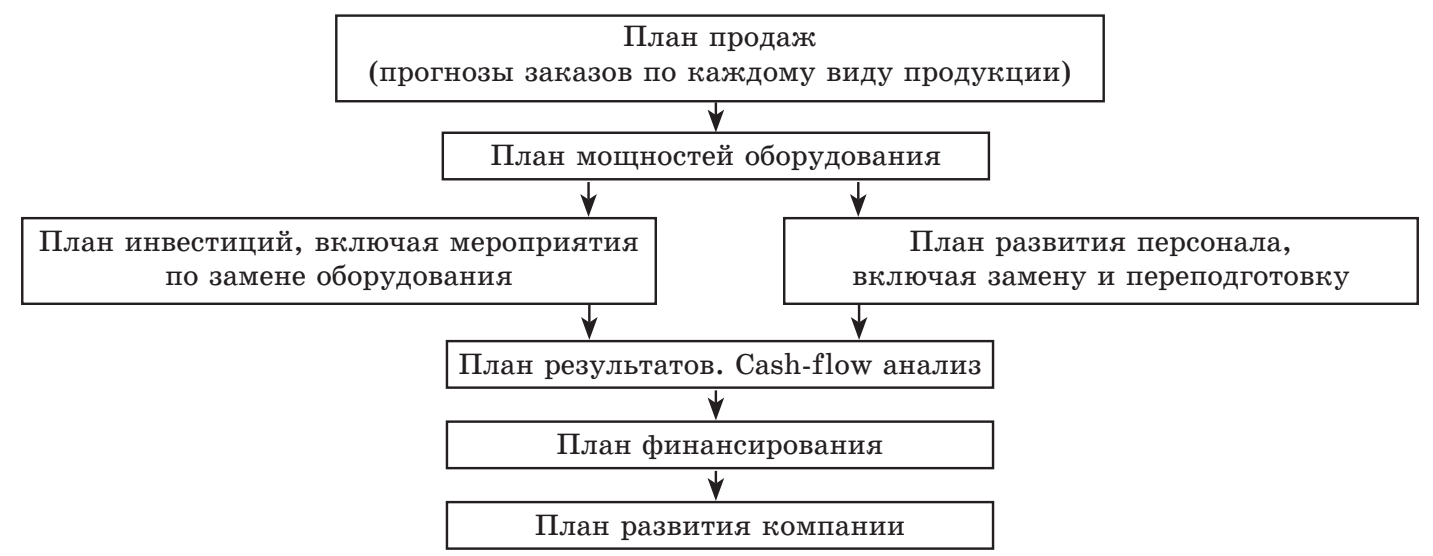

Рис. 1. Последовательность шагов в процессе лоделирования прогнозных оценок

\section{Baikal Research Journal}

электронный научный журнал Байкальского государственного университета 
Анализ и составление плана каждого из разделов схемы занимает значительное время. Однако, составив алгоритм проведения такого анализа, изменение данных о введение новых основных фондов, например, не представится таким уж сложным и займет незначительное время, поскольку матрица такого учета уже будет составлена [6].

Далее формируется раздельный аналитический учет затрат на производство в разрезе: операций передела; статей калькуляции; затрат, приходящихся на остатки заделов незавершенного производства, и затрат, совершенных в течение отчетного периода с целью распределения сгруппированных по статьям калькуляции и операциям расходов.

Загрузка ресурса на основе предлагаемого метода будет выявлена лишь в процессе совершения операции. Так, например, расчет численности рабочих на основе сложения операций по всем изделиям на конкретном рабочем месте. Этот процесс неизбежно приводит к реорганизации документооборота и оперативного учета.

В графическом виде выполняется создание диаграммы процесса с детализацией шагов по операциям от получения материалов до готовой продукции. Для операций документируется время обработки, ожидания, количество перерабатываемого материала и т.д.

Накладные расходы распределяются между различными видами продукции пропорционально сумме основной заработной платы производственных рабочих и расходов на содержание и эксплуатацию оборудования. В результате обязательно происходят неточности в исчислении себестоимости видов продукции. С развитием технологий эти неточности усугубляются, так как накладные расходы все увеличиваются, растут коэффициенты их распределения. При увеличении количества выпускаемых товаров величина накладных расходов возрастает до того момента, где перестает наблюдаться корреляция между ними и прямыми затратами труда.

Идея в том, что не надо группировать затраты по центрам их формирования, однако сделать это можно будет без проблем в выборке и формировании отчетов, если такая задача встанет на повестке дня. Затраты будут сохраняться в хранилище базы данных по каждому изделию с учетом оборудования на котором они производятся. Это и станет исходной информацией для формирования отчетов в любом разрезе, касаемо производства изделия. Учет рабочего времени и расценки также будут формироваться на операцию [7, с. 116].

Составление алгоритма прогнозных оценок деятельности компании является надстроечным инструментом, позволяющим руководителю без привлечения дополнительных работников бухгалтерии оперативно оценивать стратегические варианты. Тестовая программа явится промежуточным этапом при планировании внедрения более сложных автоматизированных комплексов.

$\mathrm{C}$ чего начинается разработка схемы автоматизации прогнозных оценок? Прежде всего - это построение «технологической линии» прохождения финансовой информации по всем этапам производственного процесса. Следующий этап - это формирование нормативной базы по принципу, заложенному еще Ф.Тейлором. Вторым теоретическим постулатом является развитие теории тойотовского канбана, который изначально работал не только без ERP, но и вообще без компьютеров [8, с. 169].

Только после решения теоретических проблем интеграции учетных политик можно приступать к разработке программного продукта в области учета движения финансов на принципах пооперационного учета с учетом неопределенности.

В заключении следует отметить, что качество учета при пооперационном методе можно увеличить точным нормированием всех производственных затрат и финансовых. Существование определенных норм дает возможность организовать

\section{Baikal Research Journal}


учет затрат на все заказы на основе нормативной системы. При данных условиях вероятен текущий оперативный контроль за израсходованием средств с фиксированием отклонений от норм.

Пооперационный метод оценки себестоимости продукции эффективен для фирм, деятельность которых характеризуется высоким уровнем накладных расходов. Снижение неточности измерений приводит к увеличению эффективности работы компании и оптимизации расходов [9].

Использование объемных показателей при пооперационном планировании может существенно изменить конечный результат расчета себестоимости продукции. Распределяя затраты по объемным показателям, при прочих равных условиях на каждое изделие независимо от его серийности, затраты на переналадку оборудования имеют одну и ту же величину, однако на одно изделие из партии в 10 тысяч приходится этих расходов в 10 раз меньше, чем на партию в тысячу изделий.

Действительная картина величины и структуры затрат позволяет компании устранить ситуацию, в которой она будет увеличивать производство малорентабельной или убыточной продукции в ущерб продукции, которая действительно является доходной, а разрабатывать и реализовывать жизнеспособные стратегии хозяйствования [10].

На сегодняшний день отчеты, полученные из различных подсистем производственного процесса в компании, проходят длительное согласование на всех уровнях организационной структуры. Снижение неточности измерений приводит к увеличению эффективности работы компании оптимизации ее расходов.

Следует отметить, что теоретические принципы бюджетного планирования деятельности структурных подразделений компании имеют значительное распространение в кругах экономистов и отказаться от этого подхода будет довольно трудно. Однако, пооперационный подход в расчете финансового плана не предполагает отказаться от подобных расчетов по существу, он ставит своей задачей учет этих расходов отдельными строчками, если это необходимо, но он, что самое главное, не ставит самоцель расчет системы бюджетов.

Бюджетная политика не может уйти от многократного использования одних и тех же исходных данных, содержания отделов аппарата управления, практически, под каждую задачу расчета бюджета. Правильный программный подход предполагает хранение информации только один раз, что исключает дальнейшие ошибки при выводе отчетов о деятельности компании.

\section{Список использованной литературы}

1. Грушина О. В. Внутрифирменное планирование в контексте системно информационного анализа процессов управления / О. В. Грушина // Известия Иркутской Государственной Экономической Академии (БГУЭП). - 2009. - № 3 (65). - С. 80-84.

2. Балдин К. В. Информационные системы в экономике : учебник / К. В. Балдин. 4-е изд. перераб. и доп. - М. : Дашков и К, 2007. - 210 с.

3. Савиных В. Н. Математическое моделирование производственного и финансового менеджмента : учеб. пособие / В. Н. Савиных. - М. : Кнорус, 2014. - 192 с.

4. Замятина O. M. Адаптация стандарта зарубежных ERP-систем к условиям современных российских предприятий / О. М. Замятина, Н. Г. Озерова // Известия Томского политехнического университета. - 2006. - № 1. - С. 193-197.

5. Цисарь И. Ф. Компьютерное моделирование экономики / И. Ф. Цисарь, В. Г. Нейман. - М. : Диалог-Мифи, 2008. - 384 с.

6. Шуплецов А. Ф. Бизнес-план фирмы. Рекомендации по разработке и анализу бизнеспроектов : учеб. пособие / А. Ф. Шуплецов. - Иркутск : Изд-во БГУЭП, 2004. - 165 с.

7. Бойделл Т. Как улучшить управление организацией: пособие для руководителя / Т. Бойделл. - М. : ИНФРА-М : Премьер, 1995. - 204 с.

\section{Baikal Research Journal}

электронный научный журнал Байкальского государственного университета 
8. Водянова Ю. И. ERP системы. Современное планирование и управление ресурсами предприятия. Выбор, внедрение, эксплуатация / Ю. И. Водяновой. - М. : Вершина, 2004. - 236 с.

9. Барановская Т. П. Информационные системы и технологии в экономике : учебник / Т. П. Барановская, В. И. Лойков. - М. : Финансы и статистика, 2010. - 310 с.

10. Самаруха А. В. Прогнозирование регионального развития при переходе России на инновационную модель экономики : учеб. пособие / А. В. Самаруха. - Иркутск : Изд-во БГУЭП, 2013. - 240 с.

\section{References}

1. Grushina O. V. Intercompany planning in the context of system-information analysis of management processes. Izvestiya Irkutskoi gosudarstvennoi ekonomicheskoi akademii = Bulletin of Irkutsk State Academy of Economics, 2009, no. 3 (65), pp. 80-84. (In Russian).

2. Baldin K. V. Informatsionnye sistemy $v$ ekonomike [Information systems in economy]. $4^{\text {th }}$ ed. Moscow, Dashkov i K Publ., 2007. 210 p.

3. Savinykh V. N. Matematicheskoe modelirovanie proizvodstvennogo $i$ finansovogo menedzhmenta [Mathematic modelling of industrial and financial management]. Moscow, Knorus Publ., 2014. 192 p.

4. Zamyatina O. M., Ozerova N. G. Adaptation of foreign ERP-systems standard to conditions of modern Russian enterprises. Izvestiya Tomskogo politekhnicheskogo universiteta $=$ Bulletin of Tomsk Polytechnic University, 2006, no. 1, pp. 193-197. (In Russian).

5. Tsisar I. F., Neyman V. G. Komp'yuternoe modelirovanie ekonomiki [Computer Modeling of Economics]. Moscow, Dialog-Mifi, 2008. 384 p.

6. Shupletsov A. F. Biznes-plan firmy. Rekomendatsii po razrabotke $i$ analizu biznes-proektov [Company's business plan. Recommendations for development and analysis of business projects]. Irkutsk, Baikal State University of Economics and Law Publ., 2004. 165 p.

7. Boydell T. Management of self-development: a guide for managers, organisations and institutions [International Labour Office], 1985. 267 p. (Russ. ed.: Boydell T. How to improve organization management: a manual for a manager. Moscow, INFRA-M, Premyer Publ., 1995. 204 p.).

8. Vodyanova Yu. I. ERP sistemy. Sovremennoe planirovanie i upravlenie resursami predpriyatiya [ERP systems. Modern planning and management of enterprise resources. Selection, implementation, operation]. Moscow, Vershina Publ., 2004. 236 p.

9. Baranovskaya T. P., Loykov V. I. Informatsionnye sistemy i tekhnologii $v$ ekonomike [Informational systems and technologies in economy]. Moscow, Finances and Statistics Publ., 2010. 310 p.

10. Samarukha A. V. Prognozirovanie regional'nogo razvitiya pri perekhode Rossii na innovatsionnuyu model' ekonomiki [Forecast of regional development in transition of Russia to an innovative model of economy]. Irkutsk, Baikal State University of Economics and Law Publ., 2013. 240 p.

\section{Информация об авторах}

Грибанова Наталия Сергеевна - аспирант, кафедра экономики предприятия и предпринимательская деятельность, Байкальский государственный университет, 664003, г. Иркутск, ул. Ленина, 11, e-mail: gribanova1992@list.ru.

Шуплецов Александр Федорович - доктор экономических наук, профессор, заведующий кафедрой экономики предприятий и предпринимательской деятельности, Байкальский государственный университет, 664003, г. Иркутск, ул. Ленина, 11, e-mail: ShupletsovAF@bgu.ru.

\section{Authors}

Natalya S. Gribanova - PhD Student, Chair of Economy of Enterprise Economy and Entrepreneurship, Baikal State University, 11 Lenin St., 664003, Irkutsk; e-mail: gribanova1992@list.ru.

Aleksandr F. Shupletsov - Doctor habil. in Economics, Professor, Head of Chair of Enterprise Economy and Entrepreneurship, Baikal State University, 11 Lenin St., 664003, Irkutsk; e-mail: ssa@isea.ru

\section{Baikal Research Journal}




\section{Для цитирования}

Грибанова Н. С. Особенности пооперационного планирования в конкурентной среде с целью оптимизации ресурсных параметров деятельности промышленной компании / H. С. Грибанова, А. Ф. Шуплецов // Baikal Research Journal. — 2017. — T. 8, № 4. - DOI : 10.17150/2411-6262.2017.8(4).12.

\section{For Citation}

Gribanova N. S., Shupletsov A. F. Features of operational planning in a competitive environment in order to optimize the resource parameters of the industrial company. Baikal Research Journal, 2017, vol. 8, no. 4. DOI: 10.17150/2411-6262.2017.8(4).12. (In Russian).

\section{Baikal Research Journal}

